ENCYCLOPEDDIE Encyclopédie berbère

BERBERE

15 | 1995

15 | Daphnitae - Djado

\title{
Dépôts rituels
}

\section{G. Camps}

\section{OpenEdition}

Journals

Édition électronique

URL : http://journals.openedition.org/encyclopedieberbere/2239

DOI : 10.4000/encyclopedieberbere.2239

ISSN : 2262-7197

\section{Éditeur}

Peeters Publishers

\section{Édition imprimée}

Date de publication : 1 avril 1995

Pagination : 2269-2276

ISBN : 2-85744-808-2

ISSN : 1015-7344

\section{Référence électronique}

G. Camps, « Dépôts rituels », Encyclopédie berbère [En ligne], 15 | 1995, document D32, mis en ligne le

01 juin 2011, consulté le 25 septembre 2020. URL : http://journals.openedition.org/

encyclopedieberbere/2239; DOI : https://doi.org/10.4000/encyclopedieberbere.2239

Ce document a été généré automatiquement le 25 septembre 2020.

(c) Tous droits réservés 


\section{Dépôts rituels}

\section{G. Camps}

1 La plus ancienne manifestation cultuelle en Afrique du Nord et peut-être dans le monde remonte au Paléolithique moyen. Il s'agit de l'amoncellement volontaire de galets dans le gisement d'El Guettar (région de Gafsa, Sud tunisien). Ce gisement, qui a livré une belle industrie moustérienne, était établi auprès d'une source artésienne. M. Gruet découvrit, au-dessous de couches tourbeuses, un curieux tas de pierres noyées dans le sable apporté par la source et qui formaient un cône régulier de $75 \mathrm{~cm}$ de haut et de $1,50 \mathrm{~m}$ de diamètre. Au sommet étaient placées quelques boules de silex; toutes les autres étaient des sphères de calcaire. Les plus petites et les plus régulières étaient dans la partie supérieure alors que les pierres de la base du tas, plus volumineuses, n'étaient que grossièrement sphériques. La plupart de ces sphéroïdes étaient naturels, quelquesuns, qui présentaient des aspérités, avaient été régularisés par piquetage. Fait notable, le fouilleur n'a pas retrouvé de tels galets dans le voisinage immédiat du gisement. Ce tas de pierre contenait également des os brisés intentionnellement et des silex taillés : 65 nucléus, 1950 éclats non retouchés et 1970 retouchés appartenant à une industrie moustérienne. Les concrétions qui soudaient entre eux les divers éléments de cet amoncellement, ainsi que le lustre très prononcé des silex et l'émoussé des os qui se trouvaient sur les bords, font penser que l'édification de ce monument fut faite en partie dans l'eau agitée et chargée de sable. 
Amoncellement volontaire de galets, de silex taillés et d'os dans le gisement moustérien d'El Guettar, région de Gafsa, Sud tunisien (Photo et fouilles M. Gruet)

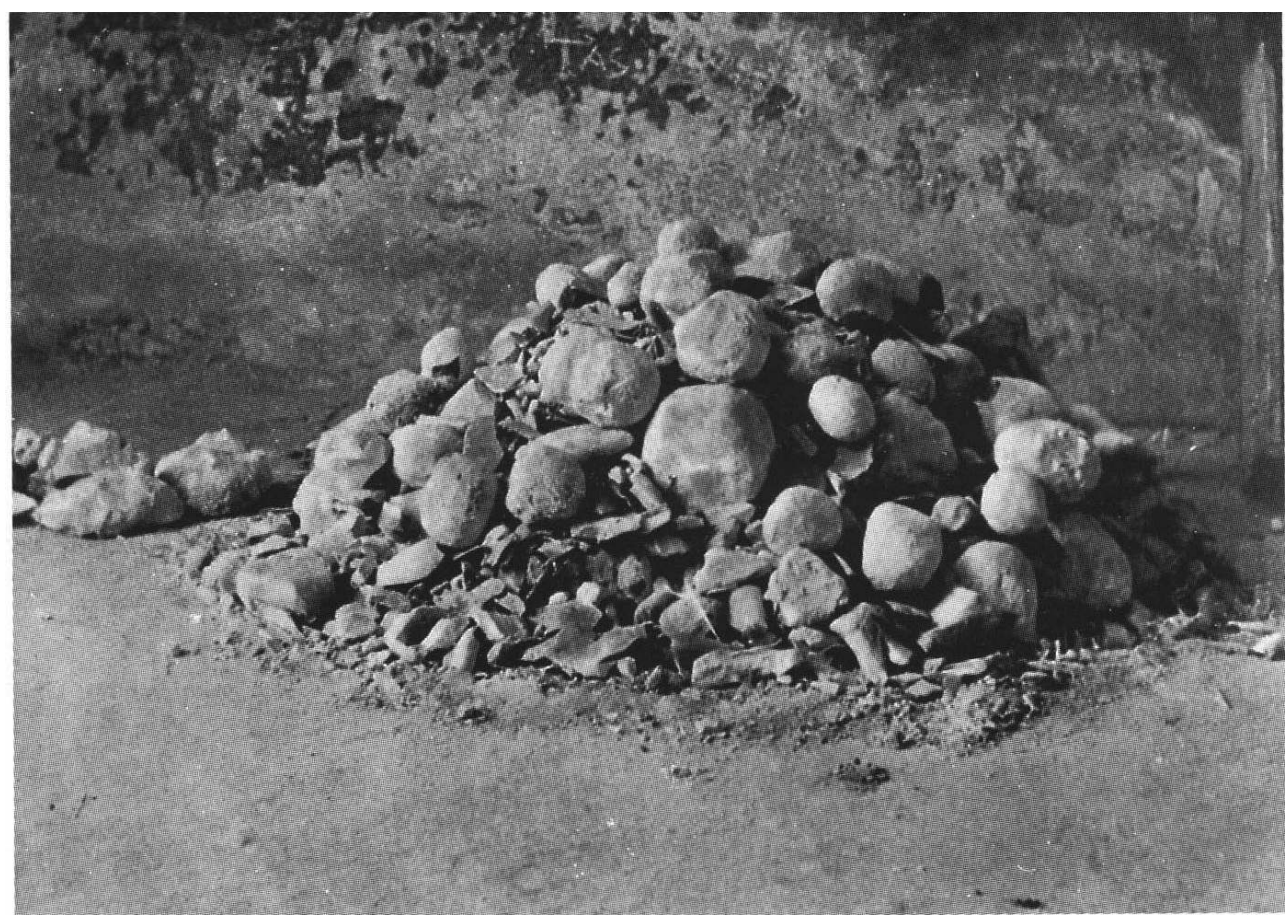

2 Il est sûr que cette structure n'obéit a aucune raison technique. Il ne peut s'agir d'une réserve de «bolas » puisque s'y trouvent intentionnellement mêlés des outils et des os en grand nombre. $\mathrm{M}$. Gruet $\mathrm{y}$ vit un dépôt votif, un cairn d'offrande à la source auprès de laquelle les Moustériens d'El Guettar s'étaient établis.

Cette manifestation des hommes moustériens d'El Guettar est, à notre connaissance, la première de pratiques très répandues dans le monde qui consistent à rassembler ou à jeter des pierres, toujours au même endroit où s'édifient ainsi de pseudo-tumulus qu'il est difficile de distinguer des tumulus funéraires sans fosse ni chambre. Il n'est pas impossible d'ailleurs que de vrais tumulus soient devenus des pierriers du fait du geste rituel qui consiste à jeter une ou plusieurs pierres sur un endroit déterminé. Quoi qu'il en soit le résultat est l'édification d'un redjem ou kerkour.

4 Ces kerkour* sont de dimensions extrêmement variables et leur répartition aussi irrégulière. Ils paraissent bien plus nombreux au Maroc, et particulièrement dans les montagnes berbères qu'en Algérie orientale et en Tunisie. Certains marquent suffisamment le paysage pour servir de limites entre des tribus. Au Maroc, certains atteignent des dimensions colossales et continuent d'être alimentés par les passants ou les pèlerins qui ajoutent leur pierre. E. Doutté cite les différentes occasions qui peuvent provoquer l'édification de tels kerkour: ce peut être la commémoration d'un acte particulier tel que la consécration d'un pacte de tad'a (voir colactation*), la mort violente d'un personnage connu ou l'abattage d'un fauve; il arrive aussi que le jet de pierres se fasse du point où l'on aperçoit une zaouïa ou le tombeau du saint auprès duquel on se rend en pèlerinage. Le plus célèbre des kerkour du Sahara me semble être le sanctuaire proche de Tazerouk (Ahaggar) connu sous le nom de Tibaradin ( les fillettes ») qui renfermait un dépôt considérable d'offrandes diverses: perles, nouets, outils préhistoriques en pierre polie et parmi eux une sculpture néolithique représentant avec un grand réalisme une tête de taureau; cette sculpture en roche 
noire était accompagnée d'une boule de quartz. Ce sont ces deux objets qui seraient à l'origine du nom donné au sanctuaire.

Il existe une autre sorte de dépôt de pierres qui est intermédiaire entre le tas de cailloux et une véritable construction; ce sont les petits amoncellements de pierres qui bordent certaines bazinas*. Ces amoncellements sont le plus souvent des autels* turriformes ruinés qui sont toujours de construction sommaire. Ces autels ont été édifiés auprès des monuments funéraires ou cultuels soit dans le prolongement du couloir d'accès ou de la niche, soit le long d'une ligne tangente à la bazina. Ces alignements peuvent compter jusqu'à une douzaine de ces autels turriformes ou de simples tas de cailloux. Il est tentant de penser que l'édification de ces autels accompagnait la visite rituelle au monument funéraire. Ces dépôts sont très nombreux dans les massifs sahariens, peut-être parce qu'ils y sont tout simplement mieux conservés en raison de la faible fréquentation de ces régions.

Dans le Tell, en revanche, les dépôts rituels les plus fréquents et les plus évidents sont les amoncellements d'objets céramiques dans de petites constructions qui, suivant les régions, reçoivent le nom de mzara ou de haouita. Les premières sont des constructions précaires de pierres plates abritant sommairement, sur des étagères de pierre ou à même le sol, de nombreux petits récipients en terre cuite. Mises à part quelques marmites ou grandes coupes (methred) dans lesquelles les femmes déposent des aliments (couscous, légumes cuits, fruits) destinés aux génies et aux pauvres, les récipients qui s'accumulent dans le petit édicule sont le plus souvent des réductions de la vaisselle domestique. Cette microcéramique est composée de godets, d'écuelles, de bols et surtout de lampes qui reçoivent plutôt des bougies que des mèches et de l'huile, sans doute trop fugaces.

Des dépôts semblables peuvent être faits à l'intérieur des haouita dont la forme la plus simple est un mur courbe dont la hauteur dépasse rarement le mètre, au centre, dans la région de plus forte concavité, est aménagée une niche dans laquelle on allume des bougies et brûle du benjouin. 
Mzara de Sidi Hamoud, région d'Ain Kebira, Algérie (Photo G. Camps)

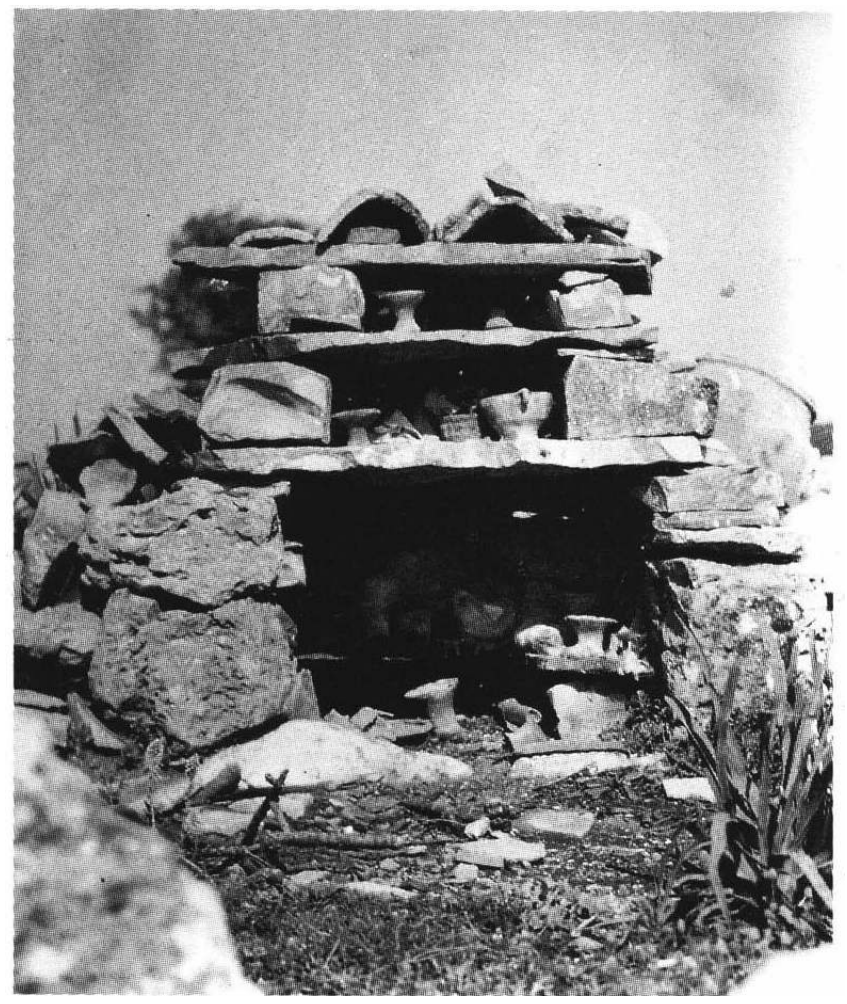

Dépôt de microcéramiques sur la tombe de Sidi Messaoud, région d'Aïn Kébira (Photo. G. Camps)

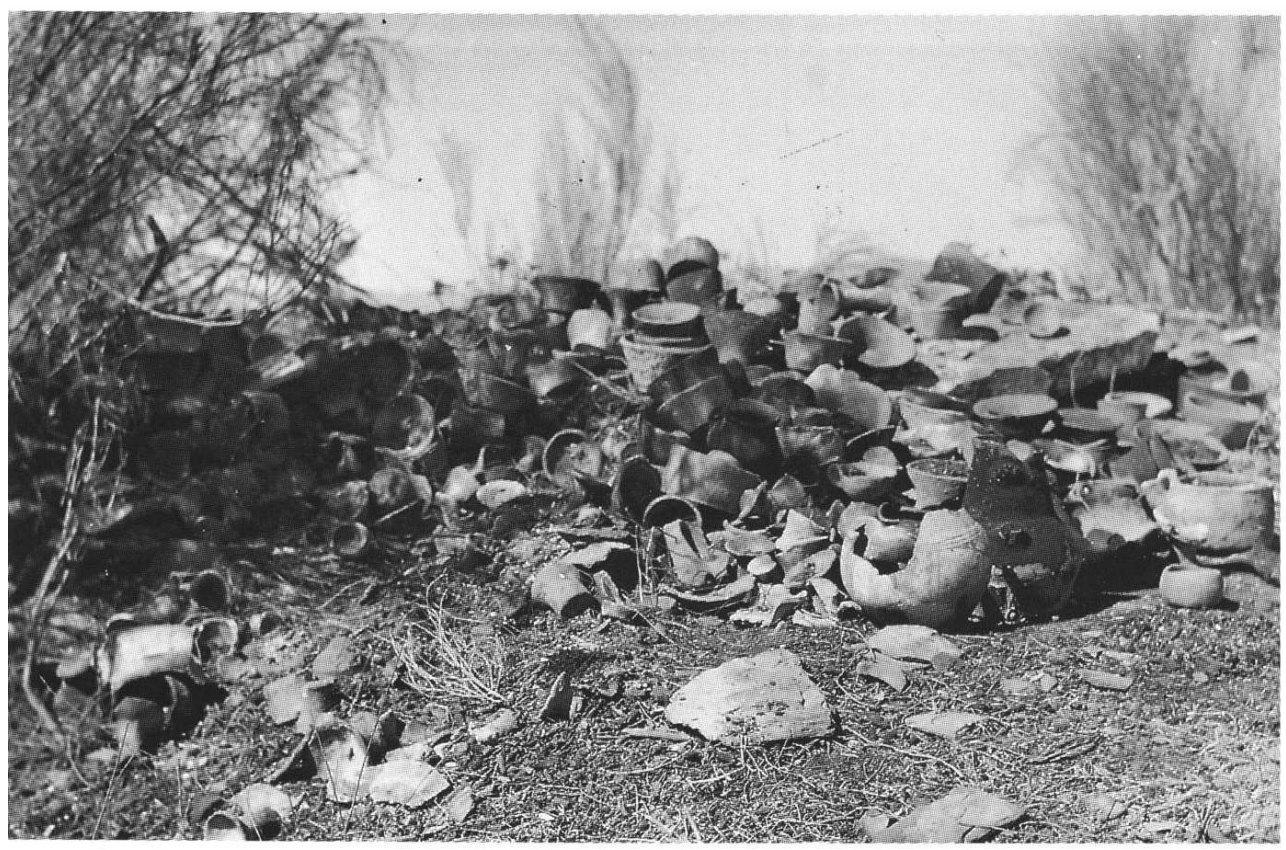

8 D'autres constructions moins sommaires reproduisent exactement la structure des koubas* (appelées à tort «marabouts »). Ce sont de minuscules monuments cubiques surmontés d'une coupole, blanchis à la chaux.

9 Les innombrables mzara, haouita, taqorabt (forme kabyle de l'arabe kouba) sont les sanctuaires les plus visibles dans les campagnes maghrébines et parfois jusque dans les 
agglomérations urbaines, mais les dépôts de microcéramique peuvent se faire en d'autres lieux moins visibles. Ils peuvent couvrir la tombe plate d'un saint présumé dont le nom n'est même pas conservé dans la mémoire collective (Sidi el Mokhfi), ou au contraire d'un personnage bien connu dont on recherche la baraka*.

10 Mais le dépôt votif peut être totalement dissocié du monde funéraire et être en relation avec celui des génies. Les sanctuaires peuvent alors être signalés au sol sous la forme d'un simple cercle de pierres ouvert généralement à l'est, ou n'être qu'une anfractuosité dans les rochers voire dans le pied d'un arbre centenaire. Il arrive que ce rocher ou cet arbre soit blanchi à la chaux, mais ce n'est pas indispensable bien que cette pratique ait été déjà en vigueur dès l'Antiquité : une dédicace latine d'Aïn Kebira (CIL, VIII, 20250) mentionne un tel blanchiment du modeste sanctuaire consacré au Numen Maurorum.

11 J.-Cl. Musso qui a particulièrement étudié les dépôts rituels des sanctuaires ruraux de Grande Kabylie, propose la classification suivante de la microcéramique qu'ils contiennent :

- les objets à feu : lampes, bougeoirs et braseros ;

- les récipients : écuelles, godets et coupes ;

- les réductions d'objets utilitaires : couvercles;

- les objets « inutilisables »: ces derniers sont surtout représentés par des cylindres de terre cuite portant à chaque extrémité une cupule dans lesquels je verrais volontiers le souvenir de balsamaires destinés à recevoir, à l'origine, quelques gouttes de parfum.

Cette typologie, valable pour la Grande Kabylie, doit être enrichie par la prise en compte des microcéramiques trouvées dans les sanctuaires d'autres régions telle que la Petite Kabylie et, d'une façon plus générale, l'Algérie orientale et la Tunisie. 
Microcéramiques déposées dans une anfractuosité de rocher à Tamanat, Anti Atlas (Photo A. Simoneau)

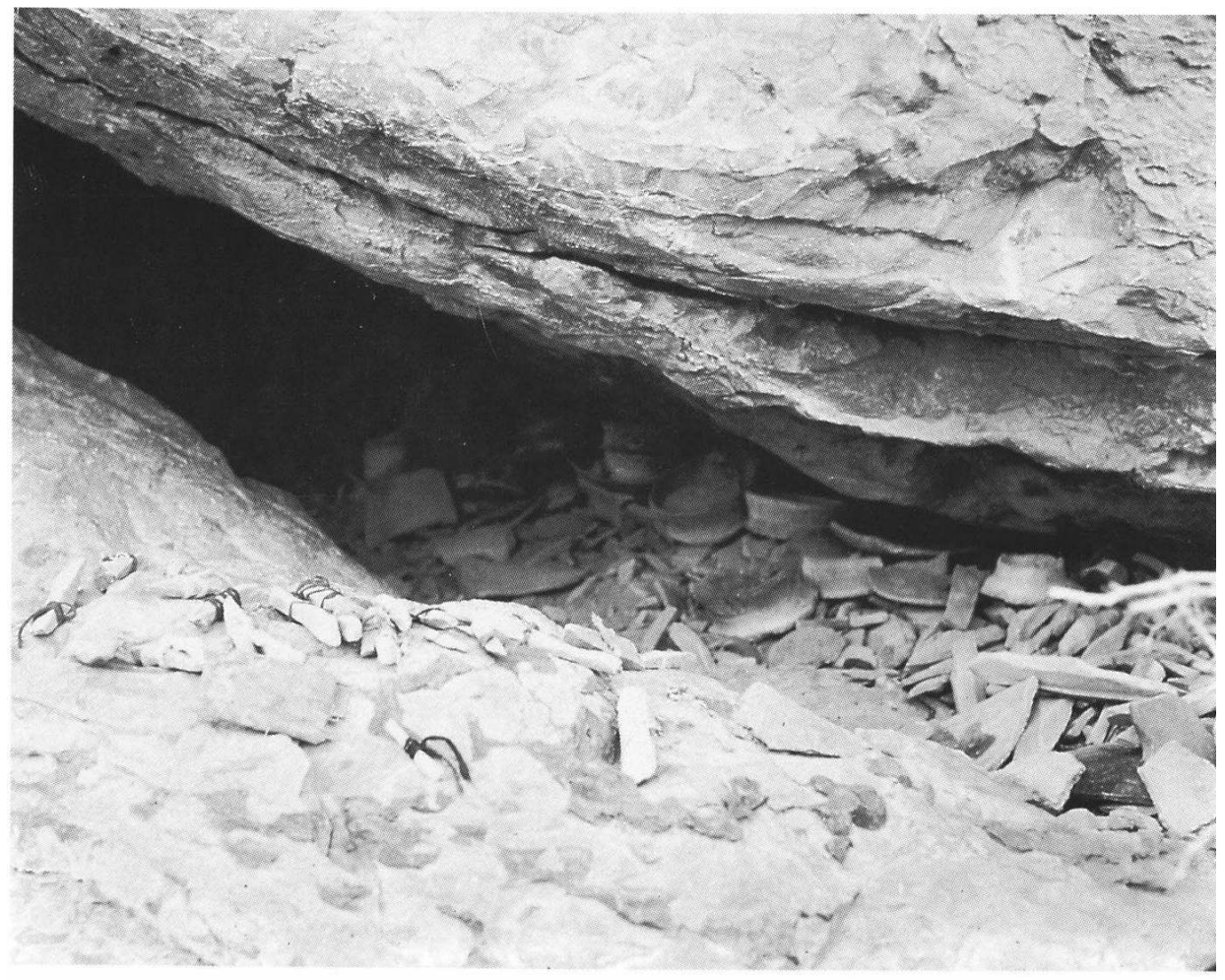

Microcéramiques et fragments de vaisselle domestique déposés entre deux strates calcaires du Djebel Mazela à Bou Nouara, Algérie (Photo G. Camps)

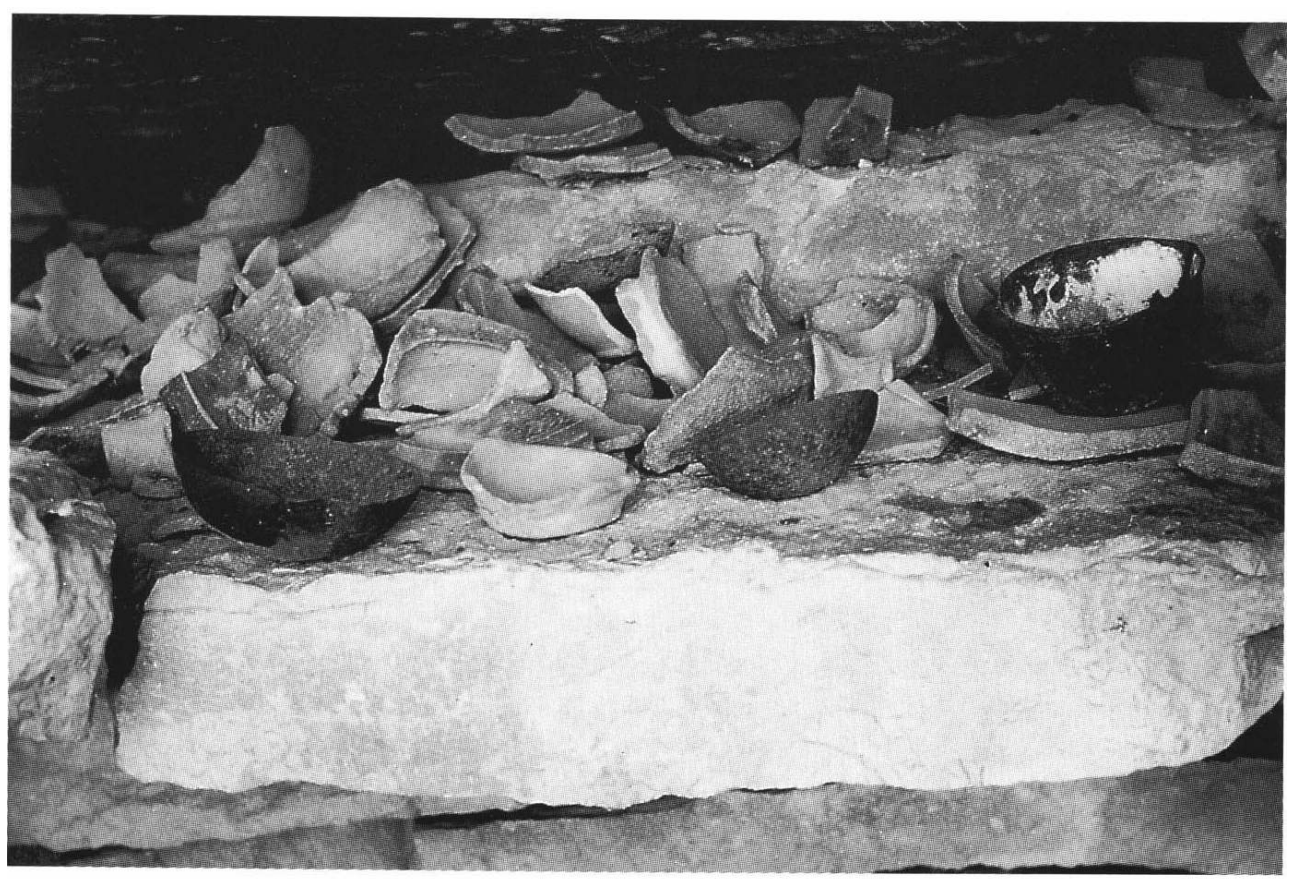

En fait on peut affirmer que n'importe quel récipient ou objet céramique de la vaisselle domestique se retrouve sous forme réduite et simplifiée dans la microcéramique votive. 
Mzara au pied d'un pistachier, région de Tizi, Algérie occidentale (Photo G. Camps)

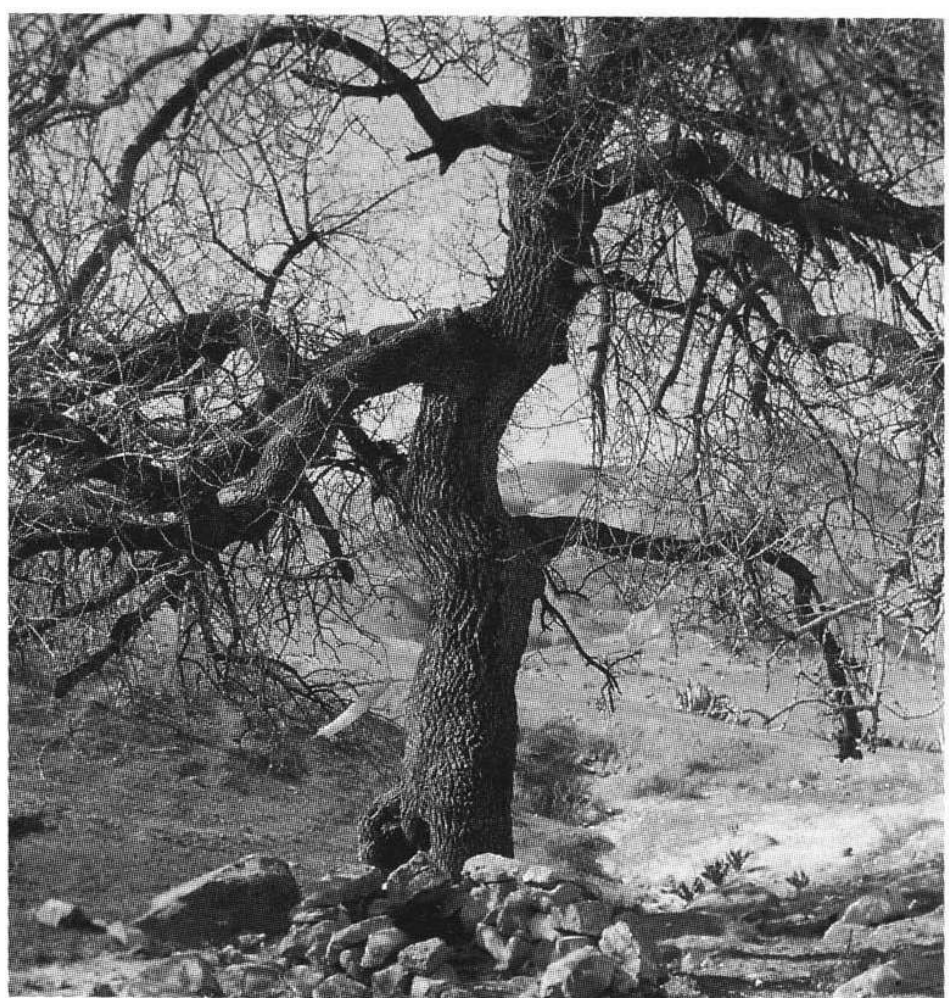

Dépôts de lampes et de bougeoirs au pied d'un olivier à Sdi Brah, Chenoua (Photo G. Camps)

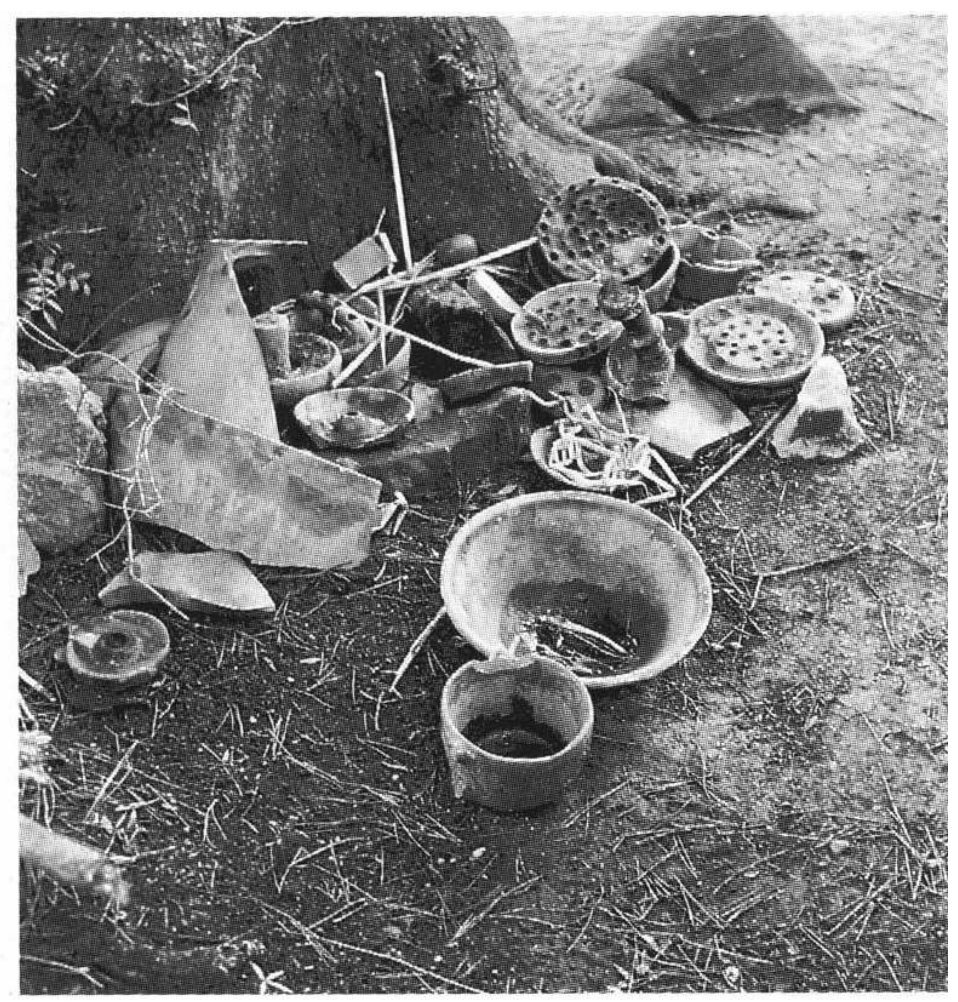

14 La plupart des microcéramiques modernes paraissent étrangement archaïques. Cet archaïsme n'est pas dû seulement à la simplicité de la technique : à part quelques 
lampes en terre vernissée achetées en ville ou quelques tasses en porcelaine ébréchées ou ayant perdu leur anse, toutes les microcéramiques sont modelées sans l'usage du tour et parmi les différents procédés connus c'est le plus primitif (abandonné dans le modelage de la vaisselle domestique) qui fut choisi pour fabriquer godets, écuelles, couvercles et balsamaires. Il consiste à modeler à pleine main une balle de pâte sans rajouter de pâte comme dans le modelage aux colombins qui est le procédé utilisé dans l'ensemble du Maghreb. Mais cet archaïsme de la technique ne suffit pas à expliquer l'extraordinaire ressemblance, pour ne pas dire l'identité qui existe entre les microcéramiques actuelles et celles qui étaient déposées dans les sépultures protohistoriques. Cette ressemblance est telle que ces objets séparés par deux ou trois millénaires sont parfaitement interchangeables.

15 Il existe bien d'autres sortes de dépôts votifs céramiques. Nous retiendrons celui de récipients de taille normale, aiguières ou cruches de formes diverses que l'on voit alignées sur les tombes ibadites du Mzab. Dans ces sépultures, la microcéramique n'est pas absente mais disposée discrètement dans des niches cultuelles aménagées dans le flanc ou à la fête de la tombe. Sur les sépultures de personnages vénérables cette niche se transforme en petit édicule et parfois en petite kouba.

Mais les dépôts rituels ne sont pas uniquement composés d'objets céramiques. Nombreux sont les seyids, marabouts ou saints inconnus dont la tombe ou la kouba reçoit en dépôt des boulets de pierre ou de fonte. On retiendra le cas du «Saint aux boulets » chez les Béni Hawa (région de Ténès, Algérie) dont la modeste tombe était signalée par deux tas de boulets en fer provenant du naufrage du Banel en 1802. Plus récemment des flotteurs de filets, sphères en verre creuses, furent aussi déposés dans certaines koubas des régions littorales.

Alignements de cruches sur des tombes mzabites à Beni Isguen (Photo G. Camps)

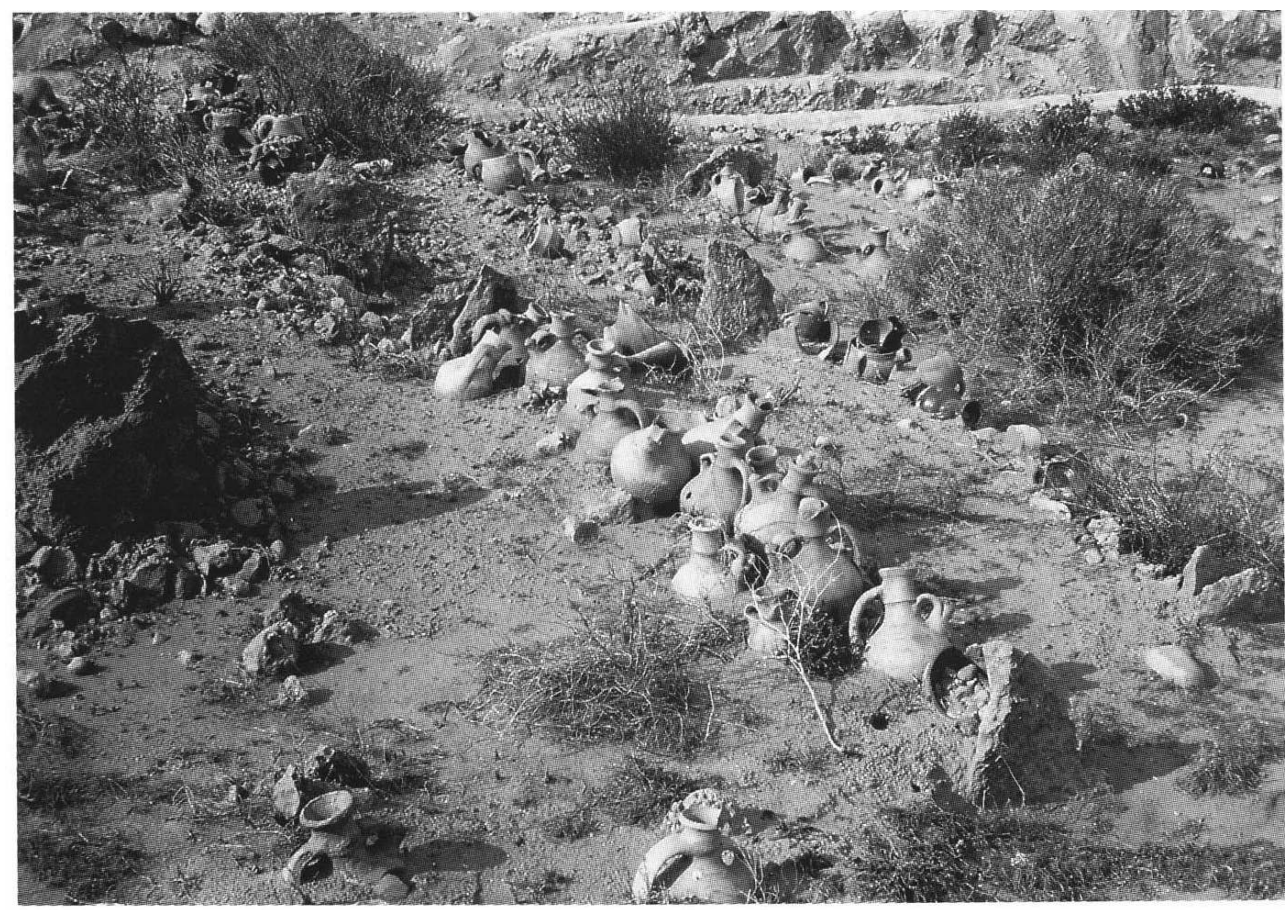

C'est à un autre rituel que répond une autre forme de dépôt aussi répandu dans le Maghreb que dans le reste de l'Ancien Monde, celui des nouets, faits de rubans ou de 
bouts de chiffons ou encore de fils de laine attachés aux rameaux d'un buisson ou d'un arbre sacré*. Les folkloristes européens se sont longuement intéressés à cette pratique déjà connue dans l'Antiquité et leurs descriptions et explications s'appliquent aussi bien au Maghreb. Je signalerai un dépôt d'aspect plus rare dans l'abri de Khelouet elBaroud (le "Sanctuaire de la poudre ") dans la région de Djelfa qui doit son nom à la pratique encore vivante qui consiste à tirer au fusil de chasse contre d'anciennes peintures dont les plus vieilles remontent à la Protohistoire. Les plombs s'écrasent contre la paroi en y laissant des traces très reconnaissables. Le support végétal faisant défaut, les nouets sont attachés à une corde fixée à la paroi, comme cela se fait aussi dans les sanctuaires rupestres de Kabylie. Les rubans et lambeaux de tissu sont accompagnés ici de fioles de parfum, de colliers et de perles de verroterie.

18 Certains sanctuaires se spécialisent dans des formes de dépôts qui leur sont originales. L'un des plus curieux que je connaisse est celui de très nombreux cadenas accumulés dans un angle du monument funéraire de Lalla Taforalt, simple construction en pierres sèches à couverture de chaume qui s'élève au sommet du Mont Chenoua* (Algérie centrale). Le dépôt d'un cadenas serait une précaution magique prise par les hommes qui émigrent en laissant leur femme au pays.

De nature très diverse, le dépôt rituel, tantôt lié au culte des saints, tantôt à celui des génies, reste, surtout dans la mentalité féminine, mais pas exclusivement dans celle-ci, une des pratiques anté-islamiques la plus répandue dans l'ensemble du monde berbère.

Nouets dans la grotte de Khelouet el Baroud, région de Djelfa (Photo G. Camps)

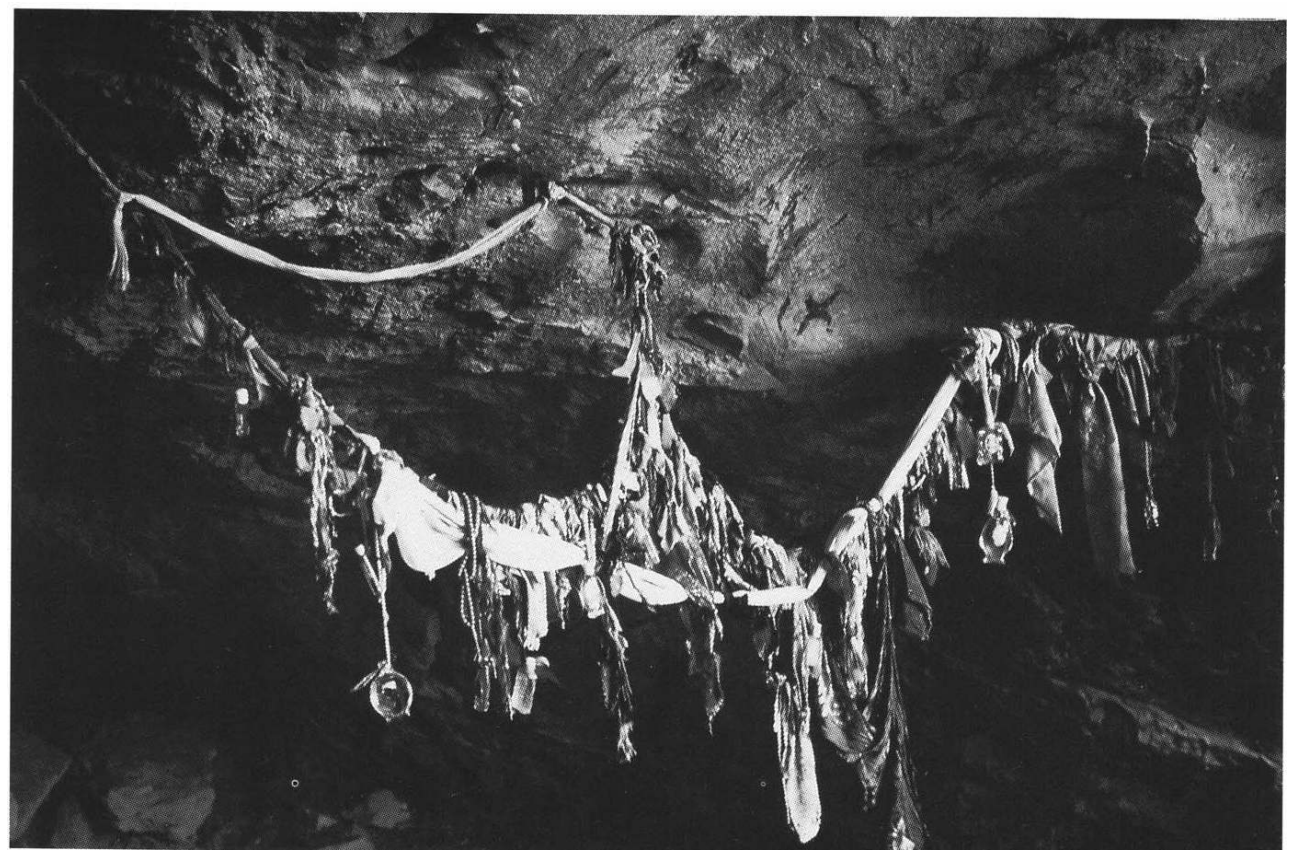




\section{BIBLIOGRAPHIE}

DOUTTE E., Les tas de pierres sacrées et quelques pratiques connexes dans le Sud du Maroc, Alger, 1903.

DOUTTE E., Magie et religion en Afrique du Nord, Alger 1908.

GSELL S., Histoire ancienne de l'Afrique du Nord, t. VI, p. 185.

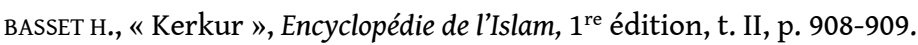

LHOTE H., « La fouille du sanctuaire des Tibaradin à Tazerouk », Travaux de l'Institut de recherches sahariennes, t. VI, 1950, p. 143-159.

GRUET M., « Amoncellement pyramidal de sphères calcaires dans une source fossile moustérienne à El Guettar (Sud tunisien) », Actes du II e Congr. panafric. de Préhist., Alger, 1952 (1955), p. 449-456.

SERVIER M., Les portes de l'Année, Paris, 1962.

CAMPS G., Aux origines de la Berbérie, Monuments et rites funéraires protohistoriques, Paris, 1962, p. 68 et 276-280.

CAMPS G, Corpus des poteries modelées retirées des monuments funéraires protohistoriques de l'Afrique du Nord, Paris, 1964.

MUSSO J.-Cl., Dépôts rituels des sanctuaires ruraux de la Grande Kabylie, Paris 1971.

AGABI C., « Colactation » Encyclopédie berbère, XIII, 1993, p. 2046-2047.

\section{INDEX}

Mots-clés : Ethnologie, Mythologie, Préhistoire, Protohistoire, Religion 